\title{
Patterned arrangement regulated mechanical properties of hydrogenated graphene
}

\author{
Yinfeng Li ${ }^{1, a, *}$, Dibakar Datta ${ }^{2,3, a}$, Shihong Li ${ }^{1, a}$, Zhonghua Li ${ }^{1}$, Vivek B. \\ Shenoy ${ }^{3, *}$ \\ ${ }^{\mathrm{a}}$ These authors made equal contribution \\ ${ }^{1}$ Department of Engineering Mechanics, School of Naval Architecture, Ocean and Civil Engineering \\ (State Key Laboratory of Ocean Engineering), Shanghai Jiao Tong University, Shanghai 200240, China \\ ${ }^{2}$ School of Engineering, Brown University, Providence 02912, USA \\ ${ }^{3}$ Department of Materials Science and Engineering, University of Pennsylvania, Philadelphia PA 19104, \\ USA \\ * Corresponding authors: Yinfeng Li (liyinfeng@sjtu.edu.cn) and Vivek B Shenoy \\ (vshenoy@ seas.upenn.edu)
}

\begin{abstract}
Molecular dynamics simulations have been performed to systematically investigate the effects of hydrogen arrangement on the mechanical properties and failure process of four hydrogenated graphene sheets with mosaic shapes (circle, square, rhombus and triangle) with same H-coverage. Compared to random hydrogenation, the interface of $\mathrm{sp}^{2}$ and $\mathrm{sp}^{3}$ bonds provided by mosaic-like hydrogen pattern retards the deterioration of intrinsic strength caused by hydrogenation. For graphene with void defects, patterned hydrogenation surrounding the void edge will
\end{abstract}


decrease edge stress. The shielding effect of hydrogenation on stress concentration provides noticeable amelioration of mechanical properties, which is sensitive to the void shape because of the difference in stress concentration. Our results suggest that the mechanical properties are tunable by manipulating the arrangement of hydrogen atoms. Patterned hydrogenation can be fully used for optimized characteristics of nanodevices fabricated from hydrogen functionalized graphene.

Keywords: Graphene; Molecule dynamics; Hydrogen functionalization; Pattern; Void defect.

\section{Introduction}

Graphene is a two-dimensional plate-like material consisting of a single layer of hexagonally arranged carbon atoms. Gifted with extraordinary thermal [1, 2], chemical [3, 4], electrical [5-7] and optical [8] properties, graphene has attracted tremendous research attention over the last few decades [9-11]. Graphene is also well known for its outstanding mechanical properties [12], which ensures it as ideal components for industry applications, such as NEMS, nanoresonators and high-sensitivity mass detectors [13]. With the advancement in the synthesis and assembly process [14], graphene can also be effectively modified via chemical addition reaction, in which the carbon atoms are converted from $\mathrm{sp}^{2}$ to $\mathrm{sp}^{3}$ hybrids to bond with the adatoms $[3,15-18]$.

Among all the adatoms or atomic clusters, hydrogen functionalization on graphene has attracted tremendous research interest not only for being a potential 
method for hydrogen storage [19], but also been acknowledged as an efficient way to manipulate the properties of graphene, such as tunable band gap [20], ferromagnetism [21] and thermal conductivity [22, 23]. The enhanced properties provided by hydrogenation are reported to be tunable by changing the hydrogen coverage [24-27]. Recent investigations also reported that arrangement of the added chemical groups including hydrogenation could affect graphene properties [15, 28-34]. To efficiently utilize such chemical functionalization with hydrogen atoms for application, it is necessary to understand the mechanical properties of the hydrogenated graphene (HG) structures $[33,35]$. Molecular dynamics (MD) simulations have been widely used to investigate the mechanical properties of $\mathrm{HG}$ with hydrogen atoms randomly distributed on the surface $[36,37]$. Recently, a successful experimental approach was reported for fabrication of mosaic graphene by patterning the doped atoms on graphene surface [34]. However, the effect of this kind of hydrogen arrangements on the mechanical properties of HG has not been fully revealed yet. There is still a gap between mechanical properties of $\mathrm{HG}$ and surface pattern of hydrogen atoms on graphene. Moreover, it is of significance to consider the mechanical properties of HG with void defects, which is unavoidable during the industrial application as well as production process [33]. Therefore, further research is in great need to reveal the effect of hydrogen arrangement on the mechanical properties of HG as well as its defective structures. In this paper, we systematically investigated this problem using MD simulations.

We first consider the hydrogenation of pristine graphene sheets, where the 
hydrogen atoms are arranged into mosaic-like patterns [34]. The mechanical properties as well as the failure process of four mosaic shapes (circle, square, rhombus and triangle) with same H-coverage are simulated, and the results show that patterned hydrogenation on pristine graphene retards the drop of Young's modulus and intrinsic strength compared to random hydrogenation. We also consider the hydrogenation of graphene sheets with void defects. Three types of void (circle, square and triangle) with the same size are designed on each pristine graphene sheets, and same amount of hydrogen atoms are hydrogenated surrounding the void. According to our results, patterned hydrogenation around the void defect will decrease edge stress on void surface. As a result, the deterioration of mechanical properties of HG caused by void defects can be ameliorated by pattern hydrogenation around void, while in sharp contrast, exacerbated by random hydrogenation.

\section{Model and method}

The simulated HG sheets have periodic boundary conditions with a lateral size of 20nm. Armchair and zigzag edges of those carbon sheets were set along the $\mathrm{X}$ and $\mathrm{Y}$ axis, respectively. We use LAMMPS package[38] for the MD simulations with an Adaptive Intermolecular Reactive Bond Order (AIREBO) potential [39] with an interaction cutoff parameter of 1.92 [36]. All the initial configurations are fully relaxed to reach equilibrium before applying uniaxial loading in $\mathrm{X}$ direction. Tensile loading with a strain rate of $0.0005 / \mathrm{ps}$ is applied by displacing the simulation box followed by a relaxation for 10,000 MD steps. The time step of our simulations is $1 \mathrm{fs}$. Such procedure of relaxation and stretching was repeated for all the HG sheets. All 
sets of the simulation were performed at room temperature under NVT ensemble. For the patterns considered in this paper, hydrogen atoms were doped on both side of the graphene sheet as shown in Fig.1. Hydrogen atoms are colored in yellow while the carbon atoms are colored in cyan. (More details can be found in SI)

We obtained the stress-strain curves by following the study of Pei et al. [36] on the mechanical properties of hydrogen functionalized graphene. The atomic volume is taken from the initial sheet after relaxation at the thickness of $3.4 \AA$. The stress of the carbon sheet was computed by averaging over all the carbon atoms in the sheet. Young's modulus $E$ and ultimate strength $\sigma$ can be obtained from the simulated stress-strain curves. The Young's modulus is calculated as the initial slope of the stress strain curve and the strength is defined at the point where the peak stress is reached.

The described MD approach was verified by studying the mechanical properties of pristine graphene ribbon with a width of $20 \mathrm{~nm}$. The simulated Young's modulus and tensile strength along armchair direction are around $\sim 0.86 \mathrm{TPa}$ and $121 \mathrm{GPa}$, which are in good agreement with experimental results of $1.0 \mathrm{TPa}$ and $123.5 \mathrm{GPa}$ [12], respectively. In the following part, the mechanical properties for each HG sheet are normalized by the properties of pristine graphene. The simulated mechanical properties for bulk graphene sheet are $E_{0}=0.82 \mathrm{TPa}, \sigma_{0}=155 \mathrm{GPa}$, which also agree with reported simulation results [40]. $E_{0}$ and $\sigma_{0}$ will be used to normalize the mechanical properties of $\mathrm{HG}$ sheets considered in the following parts. The relative difference between the effect of patterned and random hydrogenation can be directly 
revealed from the normalized results.

\section{Hydrogenated graphene with Mosaic-like hydrogen pattern}

Recently, a successful experimental approach was reported for producing mosaic graphene by patterning the doped atoms on graphene surface [34], which shed light on the fact that specific patterned hydrogenation is practical via experimental methods. Motivated by this inspiring experiment, we designed four HG sheets with mosaic-like hydrogen arrangement on the surface. As shown in figure $1,15 \%$ of the total carbon atoms of pristine graphene sheet are hydrogenated forming hydrogen domains of different geometries (circle, square, rhombus and triangle).

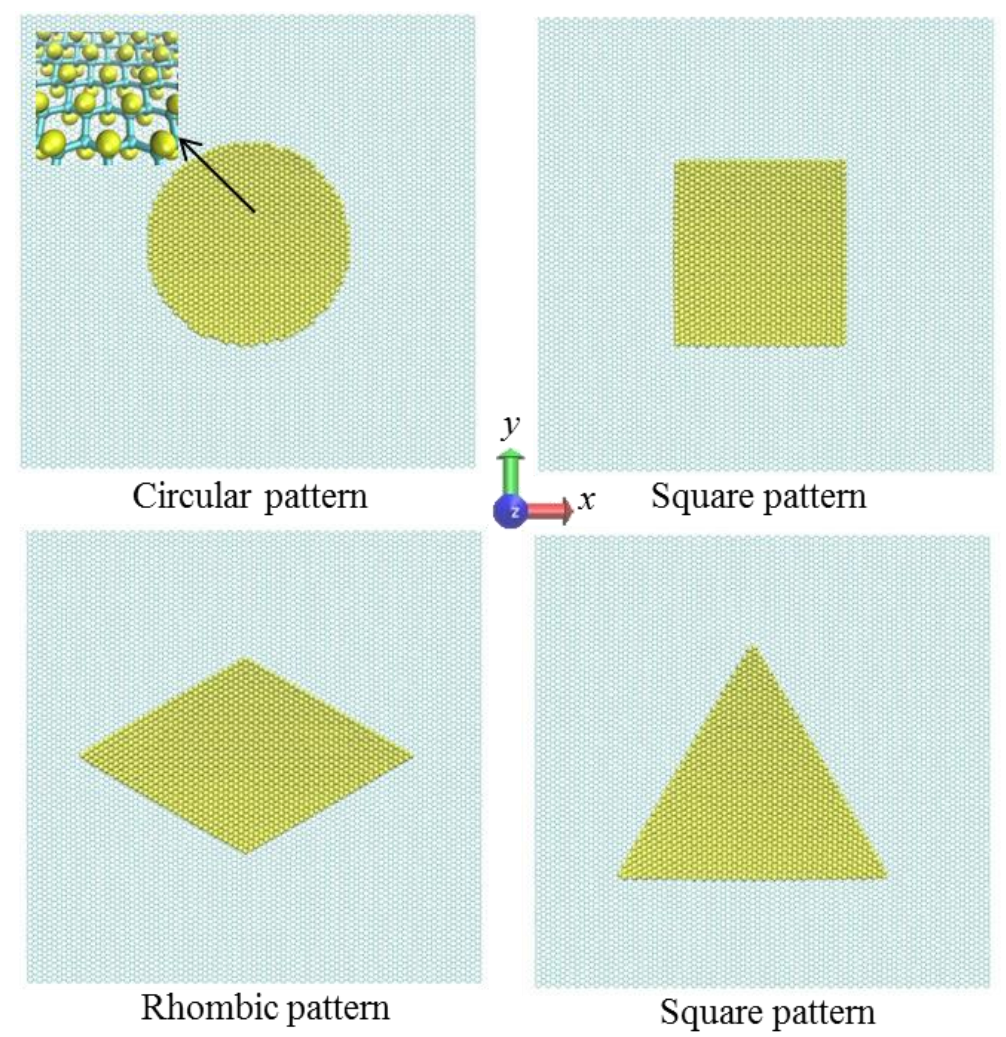

Figure 1. Hydrogenated graphene sheet with mosaic-like hydrogen arrangement on the surface. These four different mosaic-like hydrogen patterns have same $\mathrm{H}$-coverage of $15 \%$. 
As shown in figure 2, the pink line is the stress-strain curve of the corresponding HG with same hydrogen atoms randomly distributed on the surface. It can be noticed that all the patterned HG sheets have approximately the same Young's Modulus as the randomly hydrogenated one because of the same H-coverage. However, all the patterned HG sheets show higher ultimate strength and failure strain compared to that of the randomly hydrogenated one. Among all these considered mosaic-like hydrogenation patterns, the triangular pattern has the highest ultimate strength, which is $84.7 \%$ of the pristine graphene strength. The difference between the ultimate strength of patterned and unordered HG can be up to $20 \%$ of the pristine graphene strength, which suggests that the deterioration of mechanical properties caused by hydrogenation can be effectively inhibited by manipulating the physical arrangement of doped hydrogen atoms on graphene.

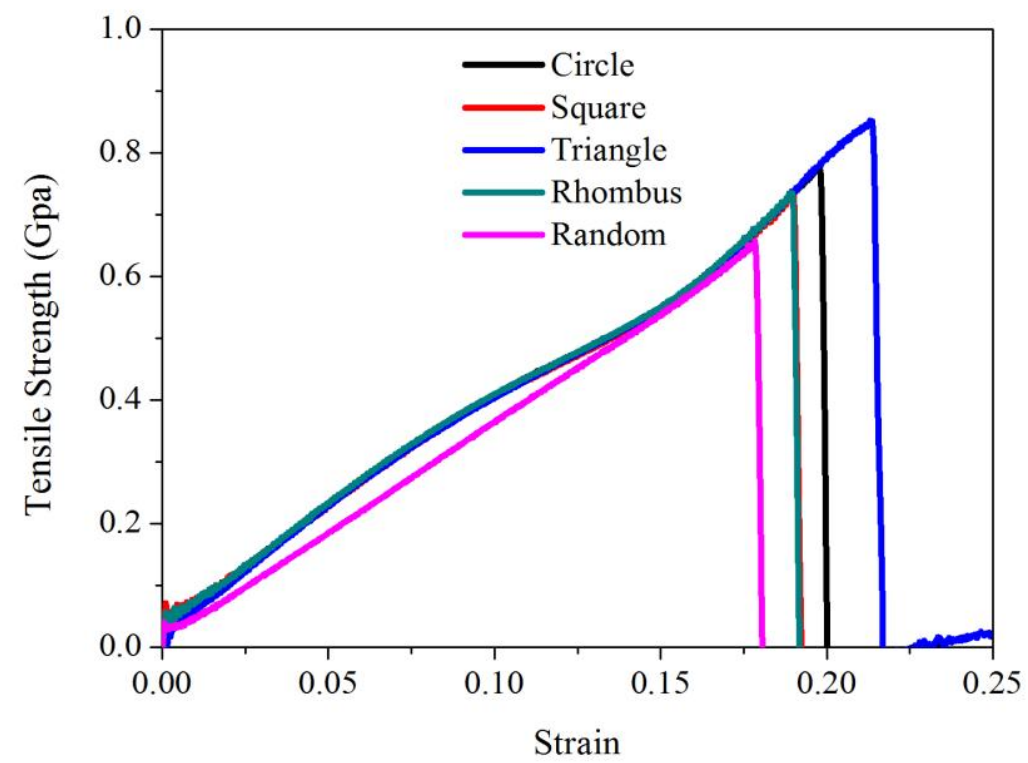

Figure 2. Stress-strain curves of four mosaic-like patterned hydrogenation and random hydrogenation (pink line) on graphene surface with same $\mathrm{H}$-coverage of $15 \%$. 
In order to interpret the physical mechanism of this interesting phenomenon, we further investigated the failure process of $\mathrm{HG}$ sheets with mosaic-like hydrogen patterns. Figure 3 shows the stress contour at the moment of bond breaking and crack nucleation for the four HG sheets with mosaic-like patterns. As reported by former investigations, hydrogenation on $2 \mathrm{D}$ carbon structures could convert the strong $\mathrm{sp}^{2}$ bond to weak $\mathrm{sp}^{3}$ bond $[36,37]$. Compared to random hydrogenation, there is an interface of $\mathrm{sp}^{2}$ and $\mathrm{sp}^{3}$ bonds on graphene provided by the mosaic-like hydrogen pattern. Under uniaxial loading, the domain with $\mathrm{sp}^{3}$ bonds has more flexibility than the surrounding domain with $\mathrm{sp}^{2}$ bonds, which behaves like a soft inclusion in matrix. As the stress contour in figure $3 a$ shows, the regions above and below the 'H-inclusion' shoulder most of the tension. The interface stress around the hydrogen domain is the result of strain mismatch at the 'H-inclusion' interface, as illustrated in figure $3 a$. It can be seen that the interface stress for circular, square and rhombic patterns are so severe that bond breaking initiated from the interface of the 'H-inclusion', followed by the vertical nucleation of crack. For the triangular pattern, the stress are more uniformly distributed around the interface of the 'H-inclusion' than other patterns, as shown in the stress contour of figure $3 a$, leading to a better ultimate strength described in figure 2. Bond breaking initiated from the $\mathrm{sp}^{2}$ domain where local stress reaches the bond limit. Due to the presence of interface stress, the crack nucleated along the triangular 'H-inclusion' interface after bond breaking. More specifically, the mosaic-like arrangement of hydrogen atoms avoids local stress concentrations, which have been reported to be the failure mechanism of HG sheet 
with randomly distributed hydrogen [36, 37].

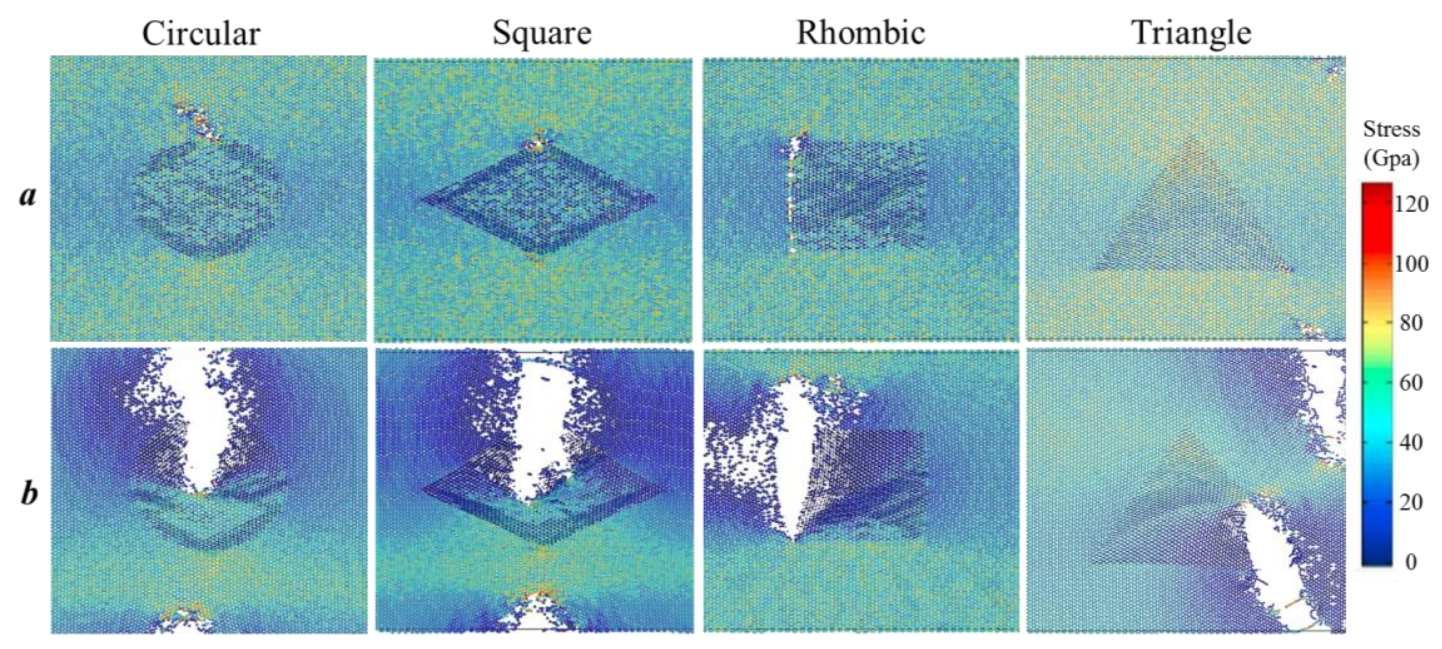

Figure 3. The stress contours of HG with mosaic-like hydrogen patterns (circular, square, rhombic and triangular) at the moment of $(a)$ bond breaking and $(b)$ crack nucleation.

We also compared the stress-strain curves of rhombic and triangular patterned HG sheets with different $\mathrm{H}$-coverage of 2, 8, 15\% (Configurations are depicted in figure S2 of the SI). Same uniaxial loading process described in figure 1 is repeated. As figure S3 depicts, the Young's modulus and ultimate strength decrease with the increase of H-coverage, which matches well with the work of Pei et al.[36].

\section{Hydrogenated graphene with void defects}

Graphene defects are usually unavoidable due to the restriction of present fabrication technique [33]. Patterned defects on graphene have been reported both by theoretical and experimental investigations [41]. Here, we will further explore the effect of hydrogenation pattern on the mechanical properties of HG with void defects.

To approach it, we first designed four HG sheets with different void shapes (circle, square, rhombic and triangle) as shown in figure 4. All these voids have the same area, 
(about $3.75 \%$ of the total area of HG sheet). The hydrogen atoms are arranged around the void to form the exact geometry as the void. All these four HG sheets have same hydrogen coverage of $11.25 \%$, i.e. the edge length of the voids is half of the corresponding outside edge of doping area. The area of hydrogen and void domains occupies $15 \%$ of the total area.

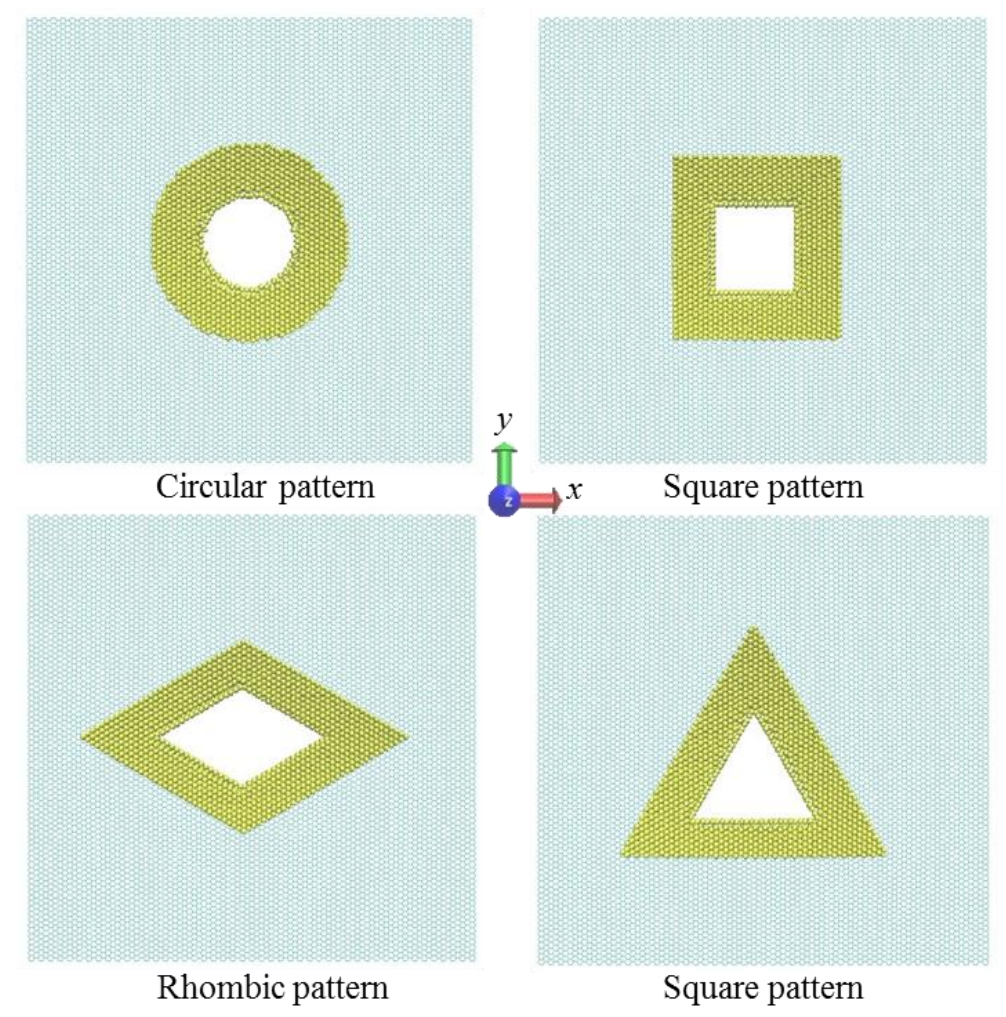

Figure 4. Hydrogenated graphene sheets with hydrogen pattern around with four typical void defects. The hydrogen atoms are arranged around the void to form the exact geometry as the void.

The black lines in figure 5 shows the calculated stress-strain curves of defective HG sheets described in figure 4. To compare the effect of patterned hydrogenation with random hydrogenation on graphene, we also consider the corresponding defective HG sheets with randomly doped hydrogen atoms, which are shown as green 
lines in figure 5. Each green line is the average of 5 different random doping. The red lines are the stress-strain curves of defective graphene sheets without hydrogenation. Figure $5 a-d$ shows the comparisons of these three graphene sheets with specific void shapes, respectively.

With the presence of void defects, the Young's modulus, ultimate strength and strain of patterned HG sheets are appreciably higher than those of randomly hydrogenated graphene sheets. It is worth noticing that the amelioration of mechanical properties is sensitive to the void shape. The properties of HG sheets with circular and square voids show high sensitivity to hydrogen arrangement. Their ultimate strength and strain of patterned structure are even $8 \%$ higher than that of the defective pristine graphene. For the cases of triangular and rhombic voids, their ultimate strength and strain of patterned HG sheet lies between that of the randomly hydrogenated graphene and defective pristine graphene.

Here, the noticeable amelioration of mechanical properties of defective HG with patterned hydrogenation is proposed to be the result of shielding effect [42] of hydrogenation on stress concentration. On hydrogenated edges, the hydrogenation around void edge will decrease the edge stress under uniaxial loading[43], which is compressive along the edge. Meanwhile, the weak $\mathrm{sp}^{3}$ bonds caused by hydrogenation provide more flexibility to the hydrogenated edge. Both the weak bond and edge stress contribute to the stress distribution in hydrogenated domain, and decrease the stress concentration on the edge. Moreover, the amelioration of mechanical properties is sensitive to the void shape because of the different stress concentration factors. For 
triangular voids, it is one order of magnitude higher than that of circular and square [44], which is much larger than the shielding effect provided by the hydrogenated edge. Thus, the optimization brought by patterned hydrogenation for $\mathrm{HG}$ with triangular and rhombic voids is less evident than those with circular and square voids.

The stress distributions of defective HG are shown in SI.
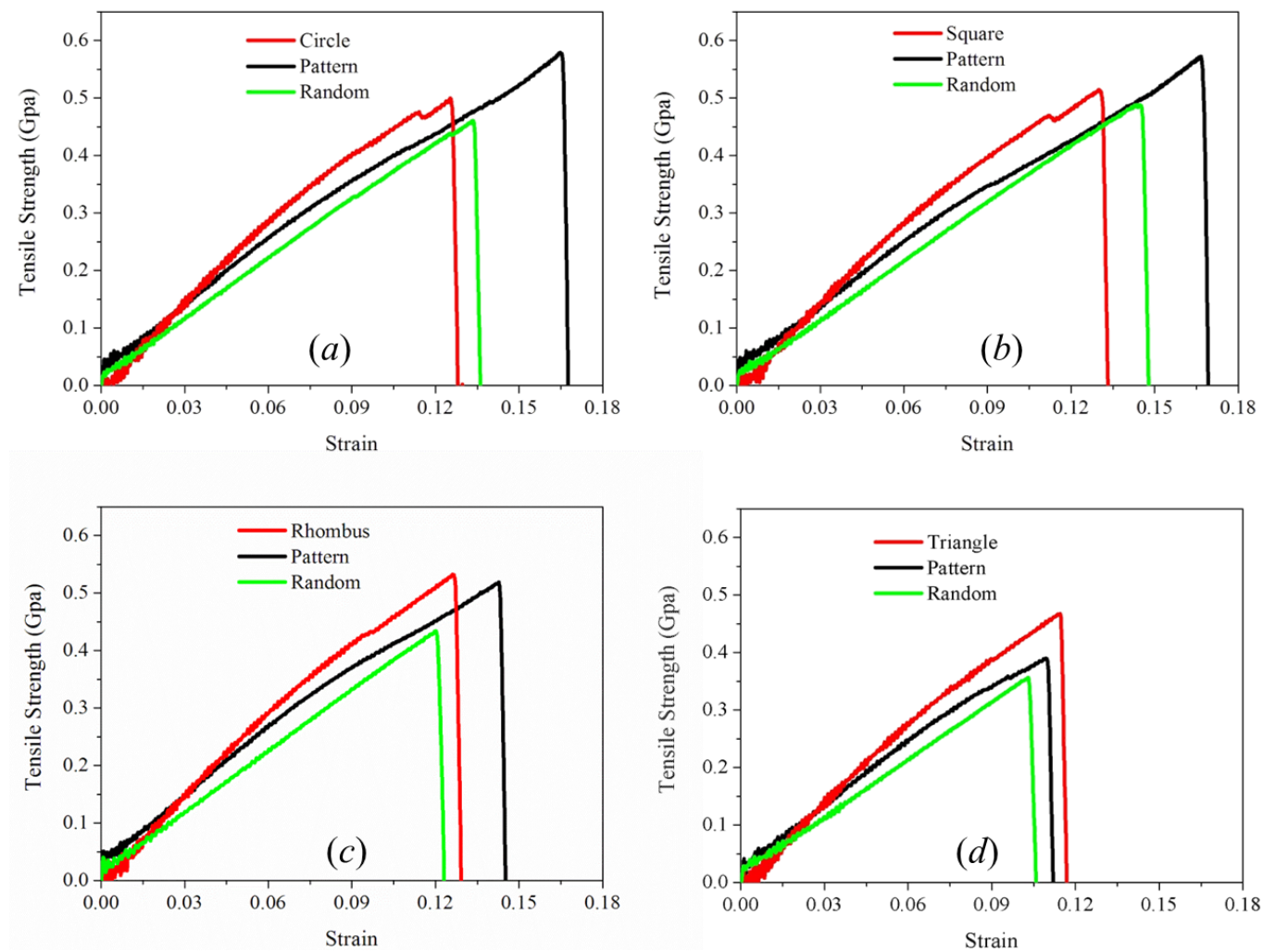

Figure 5. Strain-stress curves of the HG sheets with patterned hydrogenation around voids of different shapes (black line), as well as the corresponding graphene sheets with random hydrogenation (green line) and non-hydrogenation (red line). (a) - (d) shows the comparisons of these three graphene sheets with specific void shapes, respectively.

As an additional discussion of defective HG sheets, we design another sets of hydrogen patterns for the mechanism proposed above. As shown in figure $6 a-c$, the hydrogen atoms of three HG sheets with different voids are arranged around the void to form same circle. The solid lines in figure $6 d$ shows the stress-strain curves for 
these three HG sheets, and the dash lines are the result of corresponding defective graphene sheets for comparison. As solid lines shown in figure $6 d$, these three sheets have same Young's modulus because of the same void size and H-coverage. However, their ultimate strength and strain still show appreciably difference even with same hydrogenation pattern. The difference reveals the competition between the shielding effect of hydrogenated edge and the stress concentration of void defect. The optimization brought by the patterned hydrogenation for HG with circular void defect is larger than others because of the smaller stress concentration. For triangular voids, the optimization is less evident due to the high stress concentration at the triangular corner. The stress distributions of circularly patterned HG with different voids are depicted in SI.
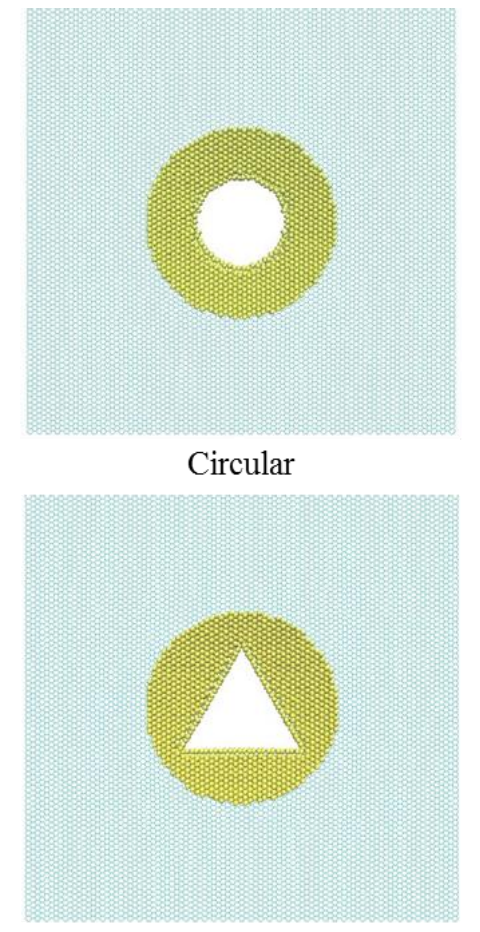

Rhombic

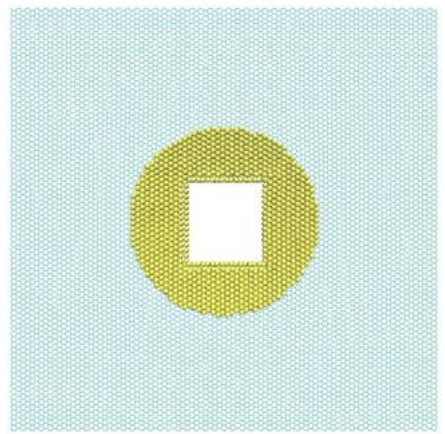

Square

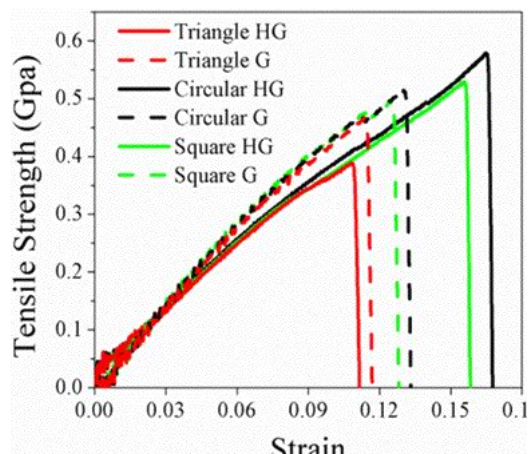

Figure 6. Comparisons of three HG with circular hydrogen pattern surrounding voids of different geometries. 
The results suggest that the stress concentration of defective HG sheet under extension could be modified by doping hydrogen atoms surrounding void edge. The shielding effect of hydrogenated edge is of great significance and potentiality when voids in graphene are required for producing NEMS equipment. The mechanical properties of defective graphene are tunable by simply adding hydrogen atoms around those holes.

\section{Conclusion}

In conclusion, we have studied the mechanical properties of specific hydrogen functionalization on pristine graphene with/without void defects by systematic MD simulations. The effect of patterned arrangement on mechanical properties is revealed by comparing with that of random hydrogenation. Our results show that patterned hydrogenation on graphene retards the decrease of mechanical properties because of the presence of interface between $\mathrm{sp}^{2}$ and $\mathrm{sp}^{3}$ bonds. The amelioration of mechanical properties when substituting random hydrogenation with patterned hydrogenation is around $10 \%$. More interestingly, for the cases of defective HG with circular and square voids, their ultimate strength, strain and Young's modulus are even higher than that of pristine defective graphene without hydrogenation. This phenomenon could be explained by the shielding effect of patterned hydrogenation on stress concentration around the void, and the optimization of mechanical properties is the result of the competition between the edge stress and stress concentration on the void edge.

Our result implies that the hydrogen arrangement pattern should be considered thoroughly before designing superior sensitive sensors, nanoresonators and other 
hydrogen storing facilities. Mastery of the effect of patterned hydrogenation on graphene will offer vital information and enlighten scientists' methods to modify, control and manipulate mechanical properties of potential applications. To enrich this topic, further research on tensile loading along zigzag direction, the anisotropic effect of patterned hydrogenation on the mechanical properties, as well as other possible geometrical patterns for different two-dimensional nanostructures is expected. Experimental verifications of our simulations are also in great need.

\section{Acknowledgement}

We gratefully acknowledge the support of the National Basic Research Program of China (No.10932007), National Science Foundation (USA), Department of Energy (USA), Army Research Office (USA) through contract W911NF-11-1-0171. Y.L. was also supported by the Open Fund of Key Laboratory for Intelligent Nano Materials and Devices of the Ministry of Education No. INMD-2014M01. The computational support for this work was provided by CCV at Brown University and MRSEC at the University of Pennsylvania. Y.L. and D.D. acknowledge helpful discussions with Dr. Junwen Li and Dr. Hemant Kumar.

\section{Reference}

[1] A.A. Balandin, S. Ghosh, W. Bao, I. Calizo, D. Teweldebrhan, F. Miao, C.N. Lau, Nano Lett., 8 (2008) 902-907.

[2] A.A. Balandin, S. Ghosh, W. Bao, I. Calizo, D. Teweldebrhan, F. Miao, C.N. Lau, Nano Letters, 8 (2008) 902-907.

[3] D.W. Boukhvalov, M.I. Katsnelson, J. Phys.: Condens. Matter, 21 (2009) 344205.

[4] A. Salehi-Khojin, D. Estrada, K.Y. Lin, K. Ran, R.T. Haasch, J.-M. Zuo, E. Pop, R.I. Masel, Appl. 
Phys. Lett., 100 (2012) -.

[5] Y. Zhang, Y.-W. Tan, H.L. Stormer, P. Kim, Nature, 438 (2005) 201-204.

[6] G. Lee, K. Cho, Phys. Rev. B, 79 (2009) 165440.

[7] D.W. Boukhvalov, M.I. Katsnelson, A.I. Lichtenstein, Phys. Rev. B, 77 (2008) 035427.

[8] L.A. Falkovsky, Journal of Physics: Conference Series, 129 (2008) 012004.

[9] V. Singh, D. Joung, L. Zhai, S. Das, S.I. Khondaker, S. Seal, Progress in Materials Science, 56 (2011) 1178-1271.

[10] M. Terrones, A.R. Botello-Méndez, J. Campos-Delgado, F. López-Urías, Y.I. Vega-Cantú, F.J. Rodríguez-Macías, A.L. Elías, E. Muñoz-Sandoval, A.G. Cano-Márquez, J.-C. Charlier, H. Terrones, Nano Today, 5 (2010) 351-372.

[11] C. Soldano, A. Mahmood, E. Dujardin, Carbon, 48 (2010) 2127-2150.

[12] C. Lee, X. Wei, J.W. Kysar, J. Hone, Science, 321 (2008) 385-388.

[13] C. Chen, S. Rosenblatt, K.I. Bolotin, W. Kalb, P. Kim, I. Kymissis, H.L. Stormer, T.F. Heinz, J. Hone, Nat Nano, 4 (2009) 861-867.

[14] G. Li, Y. Li, H. Liu, Y. Guo, Y. Li, D. Zhu, Chemical Communications, 46 (2010) 3256-3258.

[15] S. Ryu, J. Maultzsch, M.Y. Han, P. Kim, L.E. Brus, ACS Nano, 5 (2011) 4123-4130.

[16] L. Ma, H. Hu, L. Zhu, J. Wang, The Journal of Physical Chemistry C, 115 (2011) 6195-6199.

[17] F. OuYang, B. Huang, Z. Li, J. Xiao, H. Wang, H. Xu, The Journal of Physical Chemistry C, 112 (2008) 12003-12007.

[18] D.W. Boukhvalov, M.I. Katsnelson, Nano Lett., 8 (2008) 4373-4379.

[19] V. Tozzini, V. Pellegrini, Phys. Chem. Chem. Phys., 15 (2013) 80-89.

[20] J.O. Sofo, A.S. Chaudhari, G.D. Barber, Phys. Rev. B, 75 (2007) 153401.

[21] J. Zhou, Q. Wang, Q. Sun, X.S. Chen, Y. Kawazoe, P. Jena, Nano Lett., 9 (2009) 3867-3870.

[22] J.Y. Kim, J.-H. Lee, J.C. Grossman, ACS Nano, 6 (2012) 9050-9057.

[23] S.-K. Chien, Y.-T. Yang, C.o.-K. Chen, Appl. Phys. Lett., 98 (2011) 033107-033103.

[24] J. Tan, X. He, M. Zhao, Diamond Relat. Mater., 29 (2012) 42-47.

[25] L. Chen, A.C. Cooper, G.P. Pez, H. Cheng, J. Phys. Chem. C, 111 (2007) 18995-19000.

[26] X. Zhang, Y. Nie, W. Zheng, J.L. Kuo, C.Q. Sun, Carbon, 49 (2011) 3615-3621.

[27] W. Li, M. Zhao, X. Zhao, Y. Xia, Y. Mu, Phys. Chem. Chem. Phys., 12 (2010) 13699-13706.

[28] Z.F. Wang, Y. Zhang, F. Liu, Phys. Rev. B, 83 (2011) 041403. 
[29] R. Grassi, T. Low, M. Lundstrom, Nano Lett., 11 (2011) 4574-4578.

[30] C. Prachi, S.P. Bhalchandra, H. Soumyajyoti, S. Biplab, D.G. Kanhere, J. Phys.: Condens. Matter, $22(2010) 465502$.

[31] R. Balog, B. Jorgensen, L. Nilsson, M. Andersen, E. Rienks, M. Bianchi, M. Fanetti, E. Laegsgaard, A. Baraldi, S. Lizzit, Z. Sljivancanin, F. Besenbacher, B. Hammer, T.G. Pedersen, P. Hofmann, L. Hornekaer, Nat. Mater., 9 (2010) 315-319.

[32] P. Sessi, J.R. Guest, M. Bode, N.P. Guisinger, Nano Lett., 9 (2009) 4343-4347.

[33] M.Z.S. Flores, P.A.S. Autreto, S.B. Legoas, D.S. Galvao, Nanotechnology, 20 (2009) 465704.

[34] K. Yan, D. Wu, H. Peng, L. Jin, Q. Fu, X. Bao, Z. Liu, Nat. Commun., 3 (2012) 1280.

[35] R. Balog, B. Jørgensen, J. Wells, E. Lægsgaard, P. Hofmann, F. Besenbacher, L. Hornekær, J. Am. Chem. Soc., 131 (2009) 8744-8745.

[36] Q.X. Pei, Y.W. Zhang, V.B. Shenoy, Carbon, 48 (2010) 898-904.

[37] Y. Li, D. Datta, Z. Li, V.B. Shenoy, Computational Materials Science, 83 (2014) 212-216.

[38] S. Plimpton, Journal of Computational Physics, 117 (1995) 1-19.

[39] S.J. Stuart, A.B. Tutein, J.A. Harrison, The Journal of Chemical Physics, 112 (2000) 6472-6486.

[40] A. Cao, J. Qu, J. Appl. Phys., 112 (2012) -.

[41] B. Yang, J.A. Boscoboinik, X. Yu, S. Shaikhutdinov, H.-J. Freund, Nano Lett., 13 (2013) $4422-4427$.

[42] P. Zhu, L. Yang, Z. Li, J. Sun, Int. J. Fract., 161 (2010) 131-139.

[43] C.D. Reddy, A. Ramasubramaniam, V.B. Shenoy, Y.-W. Zhang, Appl. Phys. Lett., 94 (2009) -.

[44] J.-H. Woo, W.-B. Na, International Journal of Ocean System Engineering, 1 (2011) 95-101. 


\section{Figure caption}

Figure 1. Hydrogenated graphene sheet with mosaic-like hydrogen arrangement on the surface.

These four different mosaic-like hydrogenation patterns have same H-coverage of $15 \%$.

Figure 2. Stress-strain curves of four mosaic-like patterned hydrogenation and random hydrogenation (pink line) on graphene surface with same H-coverage of $15 \%$.

Figure 3. The stress contours of HG with mosaic-like hydrogen patterns (circular, square, rhombic and triangular) at the moment of (a) bond breaking and $(b)$ crack nucleation.

Figure 4. Hydrogenated graphene sheets with hydrogen pattern around with four typical void defects. The hydrogen atoms are arranged around the void to form the exact geometry as the void.

Figure 5. Strain-stress curves of the hydrogenated graphene sheets with patterned hydrogenation around voids of different shapes (black line), as well as the corresponding graphene sheets with random hydrogenation (green line) and non-hydrogenation (red line). (a) (d) shows the comparisons of these three graphene sheets with specific void shapes, respectively.

Figure 6. Comparisons of three HG with circular hydrogen pattern surrounding voids of different geometries. 


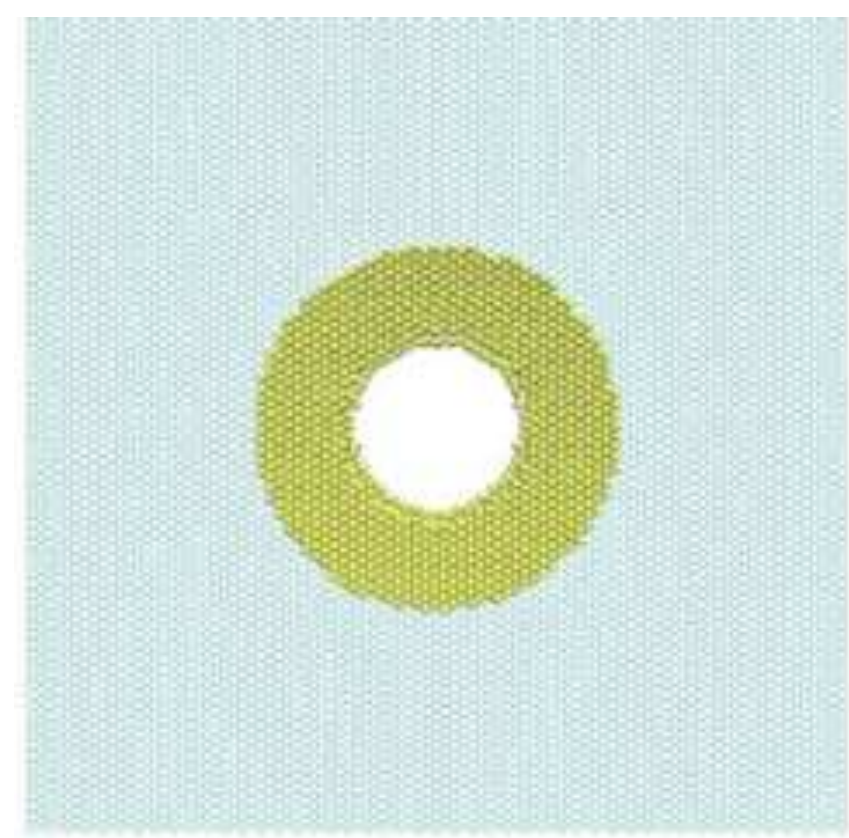

Cirentar

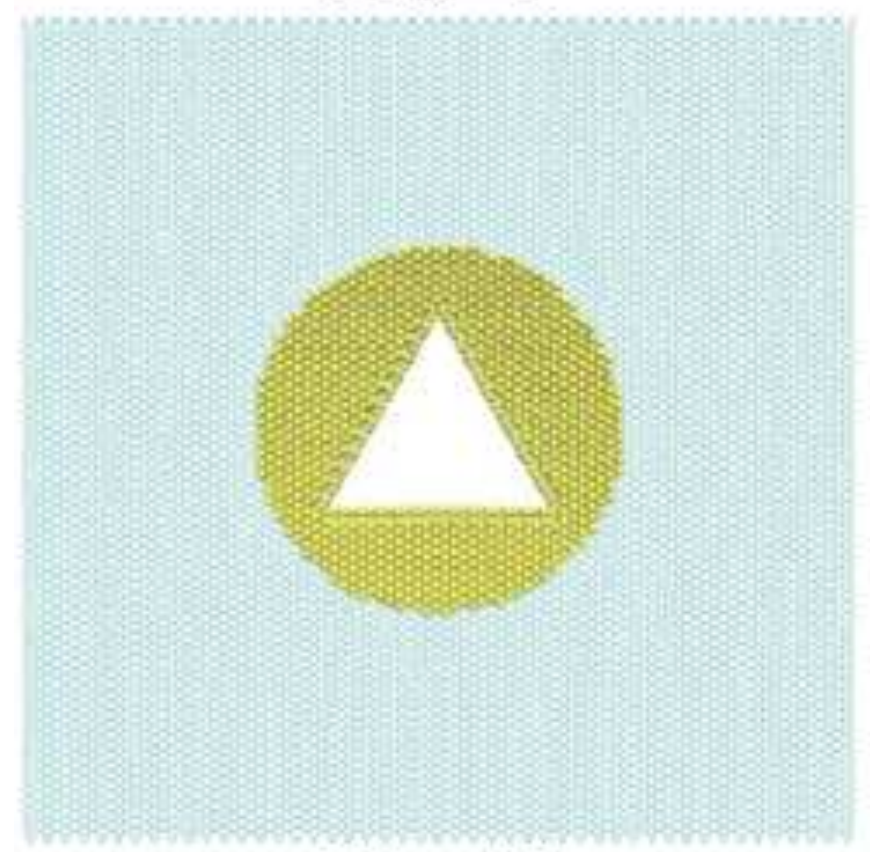

Rhombic

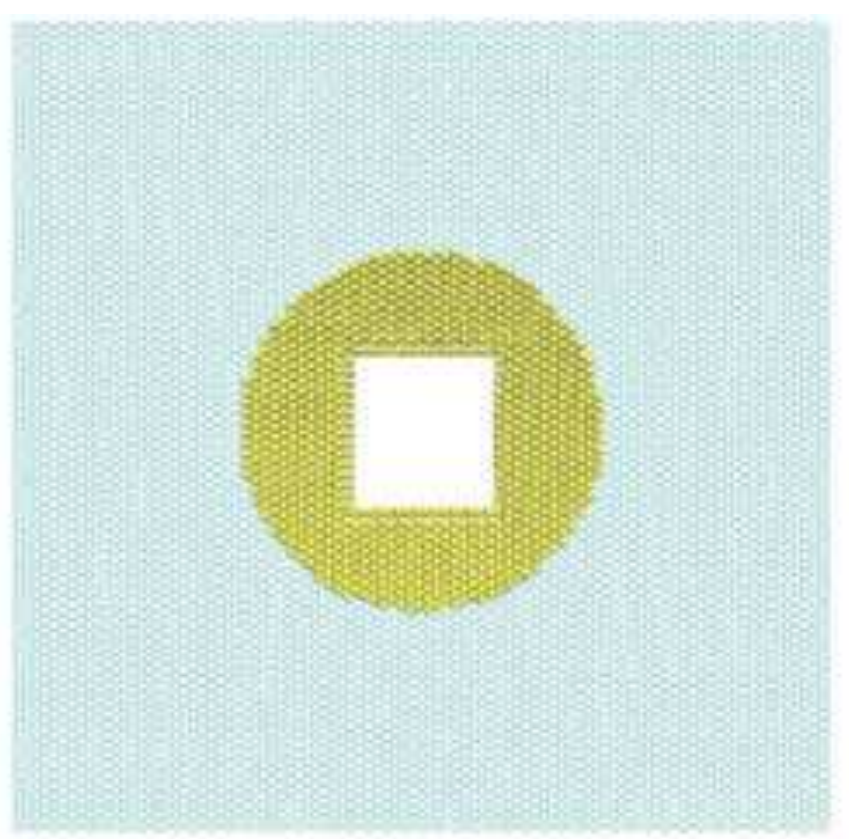

Square

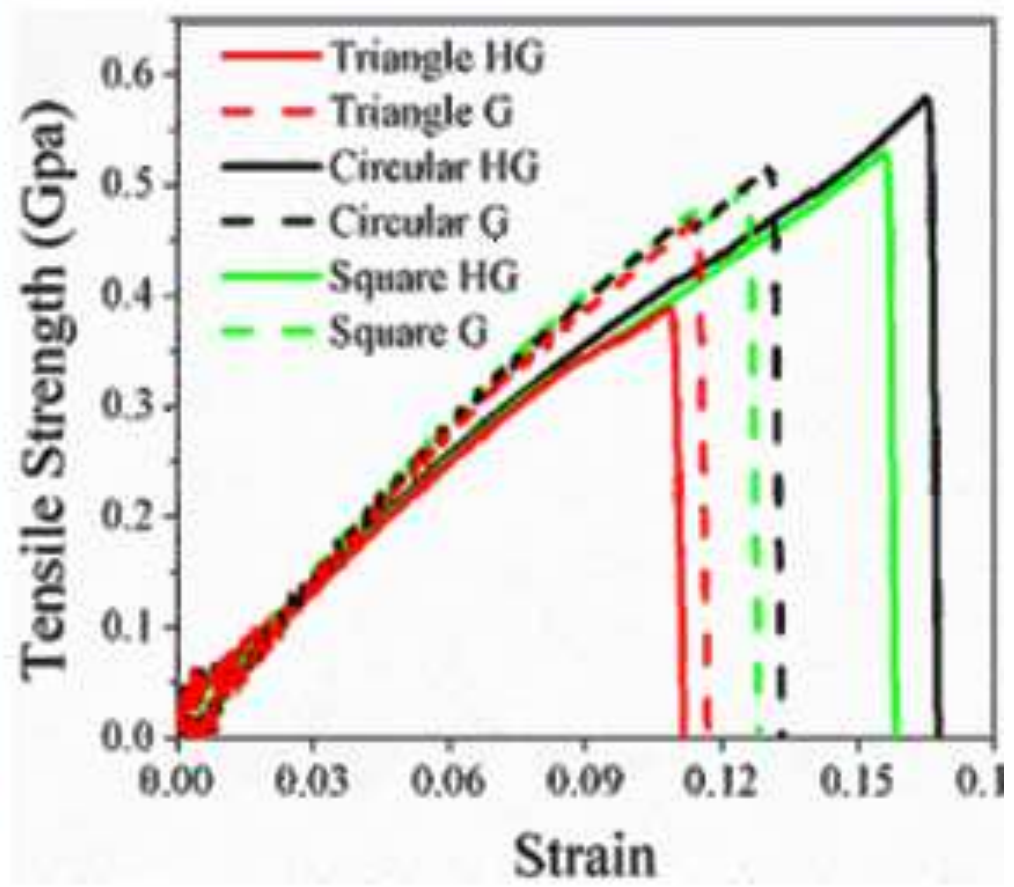

